\title{
Interesting Design of Modern Furniture
}

\author{
Di Yu \\ North China Electric Power University, Baoding 071000, China \\ 976785983@qq.com
}

Keywords: Mother Goose, Interesting Design, Furniture Design.

\begin{abstract}
Interesting design is always make people feel endless fun lead a person to endless aftertastes, design. Interesting design in an affinity, that experience in new, exciting mood, touched by the visual and emotional charm charm deeply by the work display. It can make readers enjoy the beauty in obtaining information at the same time, in the process of the aesthetic experience in the light, naturally conveys designer information.
\end{abstract}

\section{Introduction}

Papermaking technology and printing to create records of human civilization and culture of communication media, provides a visual record of information media, not only makes the design with art performance space, more far-reaching to promote the dissemination of culture and the progress of human civilization. On twenty-first Century, the global cultural difference gradually narrowed, new technology change rapidly more, the world economy integration, design in the modern cultural and commercial variations, so is the product design. Not only has an affinity for interesting design in visual communication, also can let a person produce emotional resonance in the application of product design. The combination of form, color, material and other factors and characteristics Other issues, to further explore the interesting design of humanized design in concept, bold vision of interesting design development widely and through the analysis of typical design examples of audience participation in comprehensive trend. This paper expounds the necessity of development. The development of interesting design interesting design works is the need to continue to pursue the subject.

\section{Interesting design}

\subsection{The background and concept of interesting design}

The so-called interest, are factors that can lead to some kind of affection. People can make people happy, or infected people can move people, educate people, can cause users' attention. Interest has two meanings, one is love and fun. "Love" refers to the emotion, sentiment "interest" refers to the taste. And fun. Emotion is various, there is joy, sadness. The taste is refers to the emotion in the more positive side.

The expression and artistic appeal, only constantly to spread long. Design vitality through continuous innovation in order to continue strong. Humanized design is the perfect combination of art and technology, human nature, in the design meets the basic function and the appearance, add a little attractive interest is obtained the long-term viability. In today's all kinds of design, humanized works are becoming popular. In the various elements of humanization design, interesting design in the aesthetic, emotional, cultural, the spirit more to meet the needs of the public.

\subsection{The development trend of interesting design}

Due to multiple factors of contemporary social interactions, thus contributing to the emergence of human design. The design trends and design of products is interesting and enhance the human is an important factor of development. Interesting design tend to be divided into the following three:

Firstly, the psychological impact of interesting products. A product is used in the user's hands, creates the user and the product is a kind of direct or indirect communication between designers and, no matter in which field work life, interactive psychological feelings are contemporary designers pay 
more and more attention to. The audience in the process of using the product not only completed the basic material needs, and from the heart of the feeling of pleasure. So economic growth is as can be imagined.

Secondly, the pursuit of fun products more suitable for other forms of human body structure. The development trend of contemporary and future products will be more personalized, and more suitable for the human body to use more comfortable and pleasant, and hope that the products fully integrated with the user, truly reflect the value of design.

Thirdly, mysterious interesting products and audience participation in space. The contemporary designers have to pay more attention to people's inner needs, through the design of fashion and romantic let the audience get more pleasure and intimacy. For example, there is such a wallpaper, in the elaboration, can love other own pattern and different techniques so, ordinary decorated walls and wallpaper, have alone character and story, this wallpaper to the space and the possibility of two processing, which reached a naughty and elegant effect. In the humanized design, fun design has become the inevitable trend of development, can arouse people's interest let. A person has great spiritual satisfaction and personal experience of cheerfulness.

\subsection{Interesting design innovation}

A good design works, only to be able to claim a single, concise expression, but also to attract the attention of the public, the public can be attracted and can cause the same feeling, and reached a certain drama. The design theme should be combined with the demand, is always a harmonious work, do the creative innovation again, shocking, make people resonate, think is reasonable, in contrary to expectation.

Firstly, the interesting design is also "new ideas create new styles". Franck Muler launched the design "Secret Hours" watches, to meet some people should wear beautiful watch for people to watch, and not willing to let others easily see the time of psychological needs, therefore, this watch is usually stopped at the hour and minute hand L2 position, only the second in the walk, if you want to know the time, press the button of the case at 9 o'clock, hour and minute will pinpoint the time at the moment, and then release the button can be reduced to 12 when others can see your location. The watch is beautiful, but it doesn't see the time.
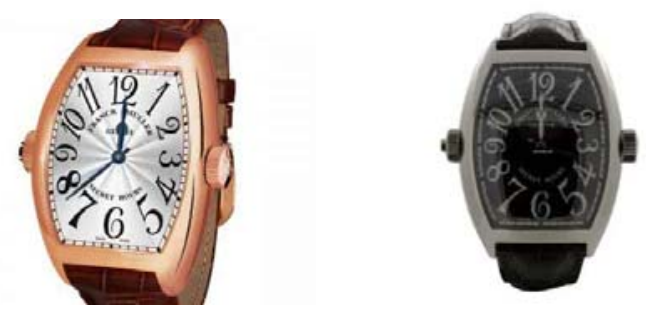

Fig. 1 "Secret Hours" watches

Secondly, beyond all expectations interesting design. "Take Your Time" is another amuse you watch style Franck Muler company design and manufacture. The dial half hour scale, 12 to 2 interval ratio of 10 to 12 or 4 to 6 of the interval to. Scale interval inequality makes people produce the illusion in visual and psychological, as if the working time will soon be over, instead of rest and recreation seemed long, although all know that is not the case, but people still feel very happy.

\subsection{Participatory design with interesting design}

On the one hand, the importance of design involvement. A work of design, when people can be involved in the further transformation, it strengthens the user's interest and stimulate the enthusiasm. For the time being called the design art design participation, the work itself is attractive, through their own creative thinking and participation, which produce changes and uncertainties in the same, it is these non qualitative factors, make the participation become infinite vitality interesting design. Modern people in the house, furniture and other stereotyped living environment to have fresh, give life to their injected new vitality.

On the other hand, participate in the design examples. For example, the "Metalarte's Lesslamp" in the first people purchase home is a complete egg shaped shell, but still need to purchase home in their own love where the tap off part of the shell, to make the light exposed so as to achieve the lighting 
effect. Because everyone focus on the different and different tools, or personal preferences of different factors, which caused the final draw completely different shape interesting effect, become a pleasure to participate in experience design, process design, has been widely welcomed.

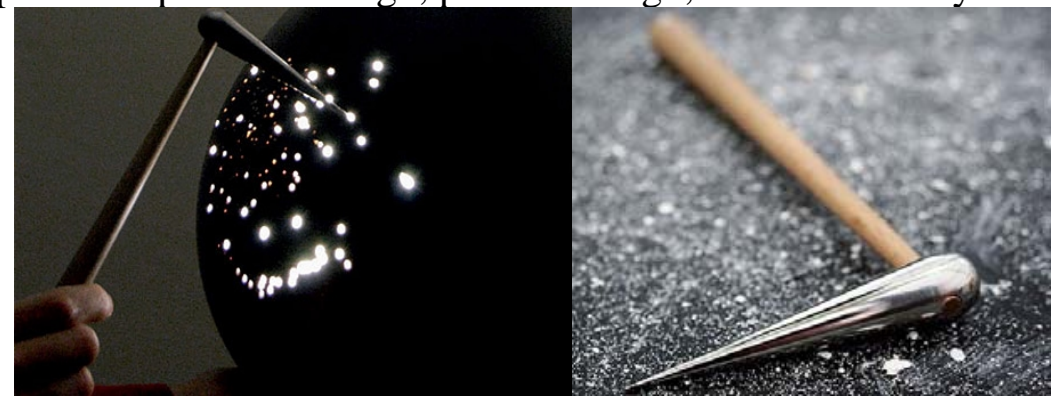

Fig. 2 Metalarte’s Lesslamp

\subsection{Method of interesting design}

\subsubsection{Modeling language}

The other language reflects the use of the product. The use of fun fun is reflected in two aspects: psychological and physiological process completely full, the overall shape of fine, steady color will give people a psychological sense of security; reasonable size, comfortable feel to happiness to people in the physical.

2.5.2 Molding material

The perceptual component increased by choosing the appropriate modeling material. Most closely with human material is biological material (such as cotton, wood etc.), followed by natural materials (stone, metal, glass, etc.), and then the non natural materials such as plastic materials. In general, the more close to the human something more cordial, a more emotional factors.

\subsubsection{Aesthetic way}

Through the study of the phenomenon of life and the relationship between the methods to explore the causes and manifestations of perceptual production. The object of beauty is often reflected in the appearance of the object is full of vitality, the vitality of the form object is actually leaked form object essence. Master creation method of object form interesting: the dynamic creation of creativity; external resistance; create growth; create connection body; break the hazy feeling, and so on.

\section{The Robin rocking chair}

\subsection{Sources of creativity-- Mother Goose}

Mother goose with the growth of content continues to expand, the inclusion of hundreds of nursery rhymes. The nursery rhymes include many bloody, cruel, the realism of the sentence, which is mainly caused by mother goose was the dark background. The impression is most songs filled with fun and warm, and never thought of these ancient rhymes and cruel existence. But the old nursery rhyme can be traced back to the historical background and social humanities style. By the dark side of nursery rhymes can let modern people know more about history. Many writers cite mother goose in his works, so many people have the opportunity to contact the mother goose, too the they are more valuable for digging.

\subsection{Product description}

The Robin rocking chair is based on the phrase "who killed Cock Robin". The rocking chair side extracts the whole line of robins, hollow design to make the whole board is not so boring, and the rope around the neck, when sitting down the rope tight, the formation of robins asphyxia visual sense; and later string is relaxed. The design of this chair is the original intention of people advocate the movement, people slumped on the rocking chair is robins is slowly destroying process. 

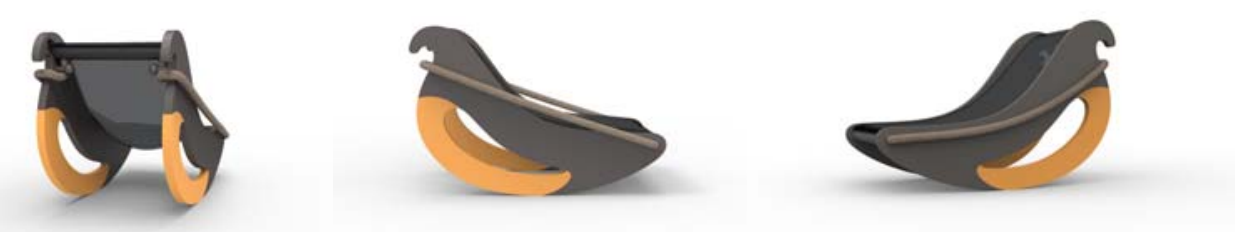

Fig. 3 The Robin rocking chair

\section{Conclusion}

Interest can make products more cordial, allowing users to smile, closer to the user. The interesting design is correct and reasonable, can increase the affinity of people and products, it can use the product itself interesting language and communication, with interesting witty or humorous to products and a non life the friendly, make a very delicate life emotion between ordinary use and by the user, that she was alive. Interesting design is brought to people's humorous atmosphere, interesting product is the embodiment of human civilization and progress. For designers, should bear some social responsibility, improve The aesthetic level of interesting products makes interest play a greater role in the stage of product design, so that the design of the product is more reasonable, more perfect and more humane

\section{References}

[1] Information on http://baike.baidu.com/

[2] Min Li, The spice design of products, [J] Art observation, (2005)

[3] Leshan Li, Ideological basis of industrial design, Beijing, China Building Industry Press,(2001)

[4] $\mathrm{Xu} \mathrm{Li}$, On the aesthetic features and manifestations of "interest", [J] Learning and exploration (2000) 